ALEJANDRA POBLETE PÉREZ

ESCUELA DE DISEÑO UTEM

SANTIAGO, CHILE

APOBLETE@UTEM.CL

\title{
Reflexiones sobre abducción, pragmatismo y diseño ${ }^{1}$
}

\author{
Abductions, Pragmatism and Design: Reflections
}

1. El presente texto corresponde a la intervención realizada por la autora en el $3^{\circ}$ Seminario Internacional "Peirce 2018. Transversalidad del pensamiento y producción del saber", organizado por los académicos del Departamento de Diseño FAU (DdD), Claudio Cortés y de la Escuela de Pregrado Jacob Bustamante, que tuvo lugar el 17 de octubre de 2018 en el Auditorio de la Facultad de Arquitectura y Urbanismo de la Universidad de Chile
Cómo citar: poblete pérez, a. (2018)

Reflexiones sobre abducción, pragmatismo y

diseño. RChD: creación y pensamiento, 3(5), 1-5

DoI: $10.5354 / 0719-837 \times .2018 .51686$
Resumen. Diseñar es establecer un nuevo orden, a partir de lo que es hacia lo que debe ser, donde este nuevo orden se expresa o materializa en un artefacto (físico o virtual). En este proceso de cambio -y toma de decisiones- están involucrados (y dosificados) componentes racionales e intuitivos. ¿Cómo se aborda el proceso?, ¿qué preguntas se hace el diseñador y cómo encuentra las respuestas?, ¿qué tipo de conocimiento es el conocimiento de y para el diseño?, ¿cómo toma el diseñador las decisiones creativas?, ¿cómo se produce el "salto creativo"?

La revisión del sistema conceptual de Charles Sanders Peirce, su pragmatismo (pragmaticismo) y su concepción de razonamiento abductivo ayudarían a la reflexión sobre aspectos esenciales que caracterizan (pero no determinan) la actividad de diseño.

El diseño, como disciplina proyectual, encuentra una afinidad epistemológica con los principios pragmatistas, según los cuales son los efectos de las cosas los que pueden tener repercusiones prácticas o experimentables y a partir de ellas se moviliza el pensamiento para la acción en el mundo real. El pragmatismo, en tanto filosofía del conocimiento, abre caminos de comprensión respecto al proceso del proyecto, así como de la investigación de diseño y su enseñanza.

Palabras clave: abducción, pragmaticismo, proceso de diseño.

\begin{abstract}
Designing is to establish a new order, starting from what it is towards what should be, where this new order is expressed or materialized in an artifact (physical or virtual). In this process of change -and decision making- rational and intuitive components are involved (and dosed). How is the process addressed? What questions does the designer ask and how do he find the answers? What kind of knowledge is the knowledge of and for the design? How does the designer take the creative decisions? produces the "creative leap"? The review of the conceptual system of Charles Sanders Peirce, his pragmatism (pragmaticism) and his abductive reasoning conception, would help to reflect on essential aspects that characterize (though do not determine) design activity.

Design, as a projective discipline, finds an epistemological affinity with pragmatist principles, according to which the effects of things are those that can have practical or experimental repercussions and from them, thought is mobilized towards action in the real world.

Pragmatism, as a philosophy of knowledge, opens ways of understanding the design process, as well as design research and its teaching.
\end{abstract}

Keywords: abduction, design process, pragmaticism.

Revista Chilena de Diseño,

RChD: creación y pensamiento

Universidad de Chile

2018, 3(5)

http://rchd.uchile.cl 


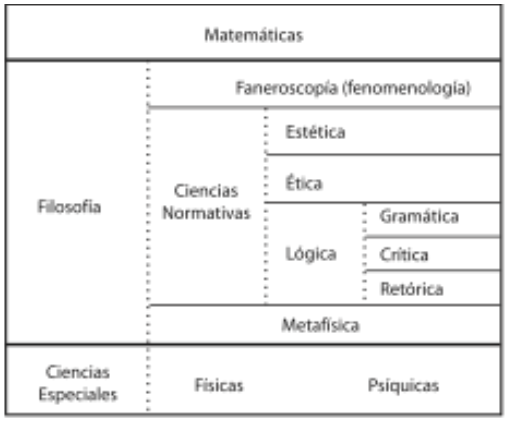

Figura 1. Extractada de Bergman, M. (2000). Meaning and mediation: Toward a communicative interpretation of Peirce's theory of signs. Helsinki: Dept. of Communication, University of Helsinki, Finland (p. 31).

2. Nota de la autora: en términos generales la epistemología es la filosofía (o teoría) del conocimiento, su definición es extensa y contiene los conceptos y énfasis provenientes de las distintas tradiciones filosóficas (alemana, inglesa, francesa o italiana). En el presente texto se ha privilegiado el enfoque anglosajón: “Es la epistemología de ámbito anglosajón y enraizada en la tradición humeana (Hume, Mill, Russell, Ayer) la que acentúa la ligazón entre conocimiento y creencia, fundamentando ambas en la evidencia. (...) Russell, en Los problemas de la filosofía, mantiene que «lo que creemos firmemente, si es verdad, se denomina conocimiento, a condición de que sea intuido o inferido lógica o psicológicamente»; y en El conocimiento humano, define asimismo el conocimiento en términos de creencia y verdad: «el conocimiento es una subclase de creencias verdaderas». Ayer, en The Problem of Knowledge, define el conocer (knowing) como «tener derecho a estar seguro»; expresión, esta última, que Gettier (1987) propone sustituir por «está justificado en creer», a fin de hacer entrar esta definición en la, según el, horma estándar. Y la Encyclopedia britanica (ed. 1961) define la epistemología como «la rama de la filosofía que se ocupa de los problemas de la naturaleza, de los límites y validez del conocimiento y de la creencia».

Actualmente los tratadistas de habla inglesa parten del supuesto básico siguiente: entre conocimiento (knowledge) y creencia (belief) hay una relación «directa» y más en concreto

\section{Introducción}

Desde un punto de vista epistemológico², el proceso del proyecto involucra aspectos deterministas e indeterministas ${ }^{3}$. Se utiliza un método (explícito o no), se estudian y analizan hechos, datos, realidades (lo que es) en el supuesto de que a partir de ello puede haber una solución. Finalmente, cuando el proceso resulta en un nuevo orden, su materialización arrojaría tantos resultados como diseñadores aborden un determinado encargo de diseño.

Por ello, un aspecto clave del proceso de diseño es: ¿cómo toma el diseñador las decisiones creativas?

¿Cómo se produce el "salto creativo" 4 ? o ¿"salto mortal sin red"s?

Al respecto, diversos autores proponen modelos (intentos deterministas) para explicar, comprender, dominar y sistematizar lo que ocurre en el proceso (todos válidos y útiles como herramientas de comprensión). Ya en 1962, en la primera Conferencia de Métodos de Diseño, en Londres, John Christopher Jones asume el componente intuitivo del diseñador reflexionando de la siguiente manera: The method is primarily a means of resolving a conflict that exists between logical analysis and creative thought (Jones, J. C.; Thornley, D. G., 1963). Es decir, la sola concepción de los, así llamados, "métodos de diseño" asume la paradoja (el conflicto, en palabras de Jones) entre lo racional y lo intuitivo, dentro del proceso del proyecto.

Es este el origen de una reflexión que encuentra puntos de contacto con la filosofía y las concepciones lógicas (teóricas) de Charles Sanders Peirce.

\section{Reflexiones en torno a Peirce}

Para entender el pensamiento de Peirce, se debe considerar que es matemático y lógico, y es desde la lógica que aborda cuestiones filosóficas, psicológicas y metafísicas. Lo central en su visión es que existen ciertas categorías universales a partir de las cuales se ordenan, en una dependencia jerárquica, las ciencias teóricas (o ciencias del descubrimiento).

La Figura 1 muestra el mapa de las Ciencias Teóricas, según Peirce.

Según el análisis que hace la investigadora Sara Barrena, su filosofía es enteramente sistemática, donde:

En lo más alto se encuentran las matemáticas, a las que pertenecen las categorías. Después de las matemáticas viene la filosofía, que tiene tres ramas principales: fenomenología, ciencias normativas y metafísica, cada una en dependencia de la anterior. Las ciencias normativas son tres a su vez: estética, ética y lógica (o semiótica), derivándose cada una de la anterior, asociadas a tres tipos de bienes (estético, ético y lógico) y que se corresponden con las tres categorías. La tarea de la metafísica sería 'estudiar los rasgos más generales de la realidad y los objetos reales'. La lógica, (...) proporcionaría la estructura para las investigaciones metafísicas, llenas de divisiones triádicas como posibilidad, actualidad, destino; azar, ley, hábito; mente, materia, evolución. (Barrena, 1996)

\subsection{Abducción y pragmatismo}

Peirce propone el concepto de abducción ${ }^{6}$ (un modo de inferencia cuyo resultado añade ideas nuevas al conocimiento) como una herramienta metodológica para la investigación científica: 
La abducción es el proceso de formar una hipótesis explicativa. Es la única operación lógica que introduce alguna idea nueva; pues la inducción no hace más que determinar un valor, y la deducción desarrolla meramente las consecuencias necesarias de una pura hipótesis. (Peirce, 1903)

Toda investigación comienza por la observación de los fenómenos, que luego son ponderados para hacer una conjetura que podría ser una posible explicación. Esa hipótesis es indispensable para iniciar una investigación, en el camino de su confirmación puede ser desechada, pero cumple una función detonadora de un proceso. Sin ella no habría proceso posible.

A través del razonamiento abductivo se llega a una conclusión que es solo probable, pero al investigador le parece momentáneamente aceptable. Ese carácter intuitivo del razonamiento abductivo es el que le otorga validez y no su efectiva probabilidad.

Para Peirce los tres tipos de razonamiento (deducción, inducción, hipótesis) son etapas de un método de indagación lógica (logic inquiry), donde la hipótesis, que denomina ahora abducción, es en realidad, la primera etapa:

La abducción es el proceso de construir una hipótesis explicativa y su forma silogística se convierte en la siguiente forma lógica:

Se observa el hecho sorprendente $\mathrm{C}$.

Pero si A fuera verdadera, C sería una cosa normal.

Por lo tanto, hay una razón para sospechar que A es verdadera.

La hipótesis se da cuando encontramos alguna circunstancia muy curiosa, que se explicaría por la suposición de que fuera un caso de cierta regla general, y en consecuencia adoptamos esa suposición. $\mathrm{O}$, cuando constatamos que en ciertos aspectos dos objetos guardan una marcada semejanza, e inferimos que se asemejan entre sí notablemente en otros aspectos.

El razonamiento abductivo es un tipo de razonamiento sintético que conjuga dos aspectos, una explicación posible y la sugerencia de una hipótesis a comprobar. Así, la noción de abducción está íntimamente ligada al pragmaticismo ${ }^{7}$ de Peirce, que en definitiva es la lógica de las hipótesis o lógica de la abducción cuyo principio es la máxima pragmática:

Consideremos qué efectos, que puedan tener concebiblemente repercusiones prácticas, concebimos que tiene el objeto de nuestra concepción. Nuestra concepción de estos efectos es pues el todo de nuestra concepción del objeto. (Peirce, 1878)

El pragmatismo es esencialmente una teoría del significado, una filosofía del conocimiento, donde conocer es hacer. En definitiva, desde la perspectiva pragmática, el conocer se origina en la interacción con el mundo, considerando al hombre como un ser activo que intenta comprender el mundo.

\subsection{Rasgos que caracterizan al pragmatismo de Peirce}

- Actitud naturalista:

El fenómeno del conocimiento es un acontecimiento del reino de la naturaleza. El sujeto del conocimiento no es un "yo pensante", sino un ser natural y social que está inmerso activamente en la realidad que investiga.

- Primacía de la práctica:

El marco general para una teoría pragmaticista del conocimiento es la actividad de los sujetos, esto es, su modo de relación con la realidad, donde el una relación de inclusión: «conocimiento es creencia de un tipo especial: creencia que satisface ciertas condiciones» (Moser y Vander Nat, 1987, 21). La cuestión central de la epistemología consiste, entonces, en determinar que condiciones debe cumplir una creencia para ser conocimiento". En Muñoz, J., \& Velarde, L. J. (2000). Compendio de epistemología. Madrid: Trotta, p. 205-206.

3. Los conceptos determinismo e indeterminismo son analizados ampliamente en Ideas sobre la complejidad del mundo (Wagensberg, 2003). Un aspecto relevante, que guarda estrecha relación con la reflexión teórica de diseño, es la concepción de determinismo en cuanto posibilidad de comprensión del mundo, la que en el texto, según el físico teórico Erwin Schrödinger, obedece al "principio de la comprensibilidad del mundo (...) la tácita hipótesis asumida desde los griegos en virtud de la cual el mundo es inteligible" (pág. 66).

4. El concepto "creative leap" (salto creativo) es utilizado por diversos autores aplicado al momento de las decisiones formales o de concreción del proyecto. Este "salto" supone un estado de "iluminación", que permite

"saltar" desde las condiciones funcionales del encargo hacia una estructura de diseño inicial del artefacto (Cross, 2007), donde es mencionado Archer, Systematic method for designers (1965), quien utiliza el mismo concepto, $y$ Maccoby The innovative mind at work (1991) con su "Model of sudden illumination". 5. En la concepción de Martí Font (1999), el

"salto mortal sin red" sería el momento de las hipótesis proyectuales. La instancia en que el diseñador, armado con el conocimiento adquirido en etapas previas "se lanza" a tomar decisiones formales, de concreción del diseño. 6. Peirce acuña los términos "retroducción" o razonamiento hacia atrás y "abducción" para referirse al proceso de adopción de hipótesis. 7. Peirce denomina Pragmaticismo a su filosofía con el objeto de diferenciarla del Pragmatismo de William James con quien no estaba de acuerdo en ciertos aspectos. En el texto, cada vez que se habla de Pragmatismo se alude al Pragmaticismo de Peirce. 
conocimiento es una estrategia, hecha de operaciones psicofísicas (sentir, entender, recordar, manipular, medir) y simbólicas (comparar, generalizar, descomponer, inferir), encaminada a la acción y coordinada con sus fines. Según esto, la acción es concebida como:

- Condición previa para el conocimiento.

- Medio para alcanzar el conocimiento.

- Resultado de la obtención de determinado conocimiento.

Así, el método, el alcance y la justificación del conocimiento obedecen a los siguientes principios:

- Empirismo:

Las "repercusiones prácticas" son efectos experimentables y, por tanto, relevantes para la acción del sujeto en algún contexto práctico imaginable. Esta es la Función Prospectiva del conocimiento (anticipación de la experiencia posible con vistas a la acción).

- Falibilismo:

La relación del individuo con su entorno es primariamente práctica y no abstracta o intelectual. Puesto que las "repercusiones prácticas" que especifican el "significado de una concepción" o concepto son potencialmente indefinidas, la verificación racional de nuestras creencias es siempre provisional y está abierta a ulterior investigación.

- Antifundamentalismo:

El conocimiento no puede reconstruirse lógicamente en la forma de una secuencia de inferencias a partir de unas primeras verdades incuestionables. La noción fundamentalista está asociada a algún tipo de garantía metafísica en relación con el conocimiento, es decir, ignora la dinámica real de las creencias y los fines de su formación y su evaluación por parte del sujeto. - Inmanentismo:

La noción de verdad (problema epistemológico central del pragmatismo), sea o no alcanzable, solo tiene sentido como un tipo de creencia, es inmanente o interna al plano de las prácticas cognoscitivas. La verdad de una creencia no puede ser independiente de su valor como guía eficaz de las acciones $\mathrm{y}$, por tanto, de su utilidad en términos de deseos y necesidades de los sujetos.

\section{Algunas implicaciones del pragmatismo de Peirce respecto al diseño} La revisión del sistema conceptual peirceano, y particularmente del pragmatismo, abre un interesante ámbito de reflexión acerca de los aspectos esenciales que caracterizan (no determinan) la actividad de diseño.

Es desde la acción (o interacción) con la realidad que surgen las tres categorías o dimensiones de esa experiencia (percepción, relación y conceptualización) y, por lo tanto, desde donde se construyen significados. Solo desde el significado es posible establecer un nuevo orden, modificar el mundo, diseñar.

De acuerdo a esta visión surgen algunas respuestas a las preguntas iniciales:

¿Cómo aborda el diseñador el proceso de diseño?

El pragmatismo, en tanto teoría del conocimiento, sitúa al diseñador frente a la complejidad, variedad y extensión del mundo, donde establecerá un nuevo orden en una posición activa y abierta para interactuar con la realidad, desde donde construirá convicciones, imaginará nuevas realidades e instalará nuevos significados. 
¿Qué preguntas se hace y cómo encuentra las respuestas?

Las preguntas surgen naturalmente de la duda y activan la generación de hipótesis. El razonamiento mediante hipótesis (abducción), aspecto clave del pragmatismo, es una eficiente herramienta metodológica (indagatoria) y epistemológica (reflexiva). Las preguntas que debe hacerse un diseñador, frente a un encargo concreto, contienen y expresan las hipótesis que movilizan el proceso de diseño, y que, paso a paso, permiten formar convicciones para tomar decisiones y concretar un determinado artefacto (proyecto). Las respuestas, en todo caso, nunca serán definitivas. Toda respuesta, traducida en una decisión de diseño puede ser replanteada por una nueva hipótesis y generar por lo tanto nuevas respuestas. Este falibilismo del proceso da cuenta de los infinitos caminos posibles del proceso de diseño, así como del aprendizaje y replanteo a partir del error.

¿Qué tipo de conocimiento es el conocimiento de y para el Diseño? Conocimiento de diseño será aquel que sirva a los propósitos del proyecto y tiene estrecha relación con las preguntas y respuestas anteriores. La misma reflexión de este artículo es parte del conocimiento de (y para) diseño y tiene que ver con un permanente "ser consciente" del proceso y sus instancias. Así también, el diseñador en su permanente objetivo de mejorar e innovar, se sitúa en una nueva y necesaria interacción (relación) con los artefactos que genera, para que desde esa experiencia, puedan surgir nuevas posibilidades funcionales (affordances), programadas por el diseñador y luego percibidas (o esperadas) por el usuario. En este sentido, las concepciones UX Design y UI Design, pueden ser entendidas como evidencias de la aproximación fenomenológica propia del Diseño.

En definitiva, el pragmatismo se hace cargo de los componentes racionales e intuitivos del proceso de investigación y propone caminos de descubrimiento cuyo valor reside en la interacción con el mundo. A partir de aquí, podrán surgir nuevas maneras de abordar tanto el proceso del proyecto como la investigación de Diseño y su enseñanza. 